\title{
Neuroendocrine-Based Cooperative Intelligent Control System for Multiobjective Integrated Control of a Parallel Manipulator
}

\author{
Chongbin Guo, ${ }^{1,2}$ Kuangrong Hao, ${ }^{1,2}$ and Yongsheng Ding ${ }^{1,2}$ \\ ${ }^{1}$ College of Information Science and Technology, Donghua University, Shanghai 201620, China \\ ${ }^{2}$ Engineering Research Center of Digitized Textile and Fashion Technology, Ministry of Education, \\ Donghua University, Shanghai 201620, China
}

Correspondence should be addressed to Kuangrong Hao, krhao@dhu.edu.cn and Yongsheng Ding, ysding@dhu.edu.cn

Received 7 June 2012; Accepted 1 August 2012

Academic Editor: Bo Shen

Copyright (C) 2012 Chongbin Guo et al. This is an open access article distributed under the Creative Commons Attribution License, which permits unrestricted use, distribution, and reproduction in any medium, provided the original work is properly cited.

\begin{abstract}
This paper presents a novel multiloop and Multi-objective cooperative intelligent control system (MMCICS) used to improve the performance of position, velocity and acceleration integrated control on a complex multichannel plant. Based on regulation mechanism of the neuroendocrine system (NES), a bioinspired motion control approach has been used in the MMCICS which includes four cooperative units. The planning unit outputs the desired signals. The selection unit chooses the real-time dominant control mode. The coordination unit uses the velocity Jacobian matrix to regulate the cooperative control signals. The execution unit achieves the ultimate task based on sub-channel controllers with the proposed hormone regulation self-adaptive Modules (HRSMs). Parameter tuning is given to facilitate the MMCICS implementation. The MMCICS is applied to an actual 2-DOF redundant parallel manipulator where the feasibility of the new control system is demonstrated. The MMCICS keeps its subchannels interacting harmoniously and systematically. Therefore, the plant has fast response, smooth velocity, accurate position, strong self-adaptability, and high stability. The HRSM improves the control performance of the local controllers and the global system as well, especially for manipulators running at high velocities and accelerations.
\end{abstract}

\section{Introduction}

With the development of the high-standard manufacturing requirement, plants become more complex while controlled by several of subchannels [1,2]. Usually, the different sub-channels have different characteristics and control requirements. Therefore, the sub-channels have to interact harmoniously and systematically to achieve multiobjective integrated control $[3,4]$. 
In that manner, the plants can have a quick start and stop, a fine uniform movement, an accurate destination, and a strong self-adaptability with stability [5]. In general, position based control cannot keep uniform velocity, while velocity-based control cannot satisfy accurate position requirement [6, 7]. It's also challenging to achieve acceleration control directly [8]. Some bio-intelligent control algorithms can overcome mathematical model problem of complex plants and have better control performances with physiological regulation to achieve multiobjective control [1,9].

Neuroendocrine system (NES) is a major homeostatic system in human body and has some outstanding multiobjective cooperative modulation mechanisms. Being a multiloop feedback mechanism, NES can still regulate the functions of several organs and glands with high self-adaptability and stability, by means of regulating their hormone secretions synchronously [10]. Some researchers have presented several models for modulation mechanism [11], feedback control [12], and hormone release [13] of NES. Based on such mechanisms, some novel artificial neuroendocrine systems (ANES) have been developed and applied to the complex control field. Neal and Timmis [14] proposed the first artificial endocrine system (AES) which includes secretion, regulation and control of hormones. The theory is applied to design a useful emotional mechanism for robot control. Vargas et al. [15] has extended the previous work of literature [14], studied the interactions between the nervous and endocrine systems and provided a comprehensive methodology to design a novel AES for autonomous robot navigation. Córdova and Cañete [16] discussed in conceptual terms the feasibility of designing an ANES in robots and to reflect upon the bionic issues highly associated with complex automatons.

To achieve multi-objective cooperative control, some recent work concentrates on how to use multi-loop and multi-objective regulation mechanism of the NES to design some novel control structures and systems. Stear [11] summarized all hormone regulation processes and described a series of control structures. Liu et al. [17] designed a NES-based two-level structure controller, which can not only achieve accurate control but adjust control parameters in real time as well. Ding and Liu et al. [18] developed a bio-inspired decoupling controller from the bi regulation principle of growth hormone in NES. Tang et al. [19] presents an NESinspired approach for adaptive manufacturing control system. Based on NES, Guo et al. [20] proposes a position-velocity cooperative intelligent controller for motor motion. Compared to conventional control system, these novel control systems always have better simplicity, practicality, stability, and adaptability. These approaches provide some new ideas to multiobjective integrated control field and have good results in simulation. Nevertheless, no experiment has been done on actual plants, especially for multi-objective cooperative control of the position, velocity and acceleration of different parts of the controlled plant.

In this paper, a novel multi-loop and multi-objective cooperative intelligent control system (MMCICS) based on regulation mechanism of NES is proposed. Inherited from NES, the MMCICS consists of four subunits: Planning unit regulates position, velocity and acceleration signals based on ultralong loop feedback. Selection unit is a soft switcher to smoothly select dominant motion control signal based on long loop feedback. As short loop feedback, coordination unit is responsible for processing and transmitting coordination signals to several sub-channels in execution unit. The execution unit is an integrity whose sub-channels interact harmoniously and systematically based on ultra-short loop feedback. Each channel has a proposed hormone regulation self-adaptive module (HRSM) which identifies control error and regulates control parameters in real time. The control performance of the proposed MMCICS is verified by an actual 2-DOF redundant parallel manipulator. The experimental results demonstrate that, through regulation mechanism of the MMCICS, the multiobjective 
integrated control task can be achieved easily while the stability, accuracy, adaptability, and response rate of the plant is improved by proposed HRSM.

The main contribution of this paper lies in that it generalizes the characteristics of the NES for regulation, and then reveals the similarity between the NES and a motion control system where the coordination of position, velocity, and acceleration are implemented by the cooperation of different subchannels of the plant. Furthermore, based on the regulation characteristics of the NES, a bioinspired motion control approach is provided, it has been used in MMCICS design. According to our knowledge, this is the first time that the MMCICS based on biological NES is proposed and especially applied to an actual manipulator. The proposed approach is practical and easy to implement, which provides a new efficient method for the intelligent control of complex systems.

The remainder of this paper is arranged as follows. In Section 2, the regulation mechanism of the NES is described while a corresponding bio-inspired motion control approach is presented. In Section 3, the detailed design of the MMCICS is elaborated including system structure, control algorithms, and parameters tuning methods. The experimental results are given to verify the effectiveness of the proposed control system in Section 4. Finally, the work is summarized in Section 5.

\section{Regulation Mechanism and Bioinspired Motion Control Approach}

\subsection{Regulation Mechanism of Neuroendocrine System}

The NES mainly includes nervous system and endocrine system [15]. The nervous system is primarily responsible for receiving stimuli of environmental change and processing corresponding nerve impulse. The endocrine system can be viewed as a system of glands that works with the nervous system in regulating the activity of internal glands and coordinating the long-range response to external stimuli [21]. One of the most important interactions between them is regulated by means of their hormone secretions.

A typical regulation mechanism of the neuroendocrine hormone can be generalized as follows $[12,13,20,22]$ : central nervous system detects the changes in the internal and external environments and transmits the nerve impulse as appropriate response to hypothalamus. Hypothalamus receives the nerve impulses and secretes relevant releasing hormone $(\mathrm{RH})$, which stimulates pituitary to secrete tropic hormone (TH). Under the influence of pituitary's $\mathrm{TH}$, other glands (such as thyroid, adrenal, gonads, etc.) secrete corresponding hormones which regulate the situation of human physiological balance. There are massive of feedback loops in neuroendocrine system. Four types of typical feedbacks include ultra-short, short, long and ultra-long loop feedbacks $[22,23]$. The ultrashort loop feedback means that the hormone released by a certain gland is directly fed back to its source and changes its status. In the short, long and ultralong loop feedbacks, the concentration of corresponding hormone is fed back to the pituitary, hypothalamus and central nervous system, respectively. Through the multiloop feedback mechanism, multihormone control is stable and easy to practice, as shown in Figure 1.

\subsection{Regulation Characteristics and Bioinspired Motion Control Approach}

The regulation characteristics of NES can be summarized as below: (1) the NES has several feedback loops and glands. Each feedback mechanism has its own function and different 




Figure 1: Hormone regulation of the NES.

messages can be transferred among them so that the whole system has a multiobjective regulation mechanism of integrity. (2) Central nervous system is the foremost command center. (3) Hypothalamus is the medium between the nervous system and the endocrine system. (4) Pituitary has the ability to achieve multi-hormone coordinative control. (5) The different glands always have different hormone secretion scopes and different hormone secretion standards. But they have the similar regulation mechanism that can enhance identification and secretion precision within a certain range of stimulus [13].

Therefore, corresponding to the motion control system, the central nervous system, the hypothalamus, the pituitary, and glands of NES can be regarded as the planning unit, the selection unit, the coordination unit, and the execution unit, respectively. In this scenario, the planning unit receives input signal and transmits the suitable motion planning signal to the selection unit. The selection unit processes the motion planning signal and chooses the dominant motion control signal. And then, the coordination unit converts dominant motion control signal to various coordination signals according to its performance characteristic. Various sub-channels in the execution unit receive their own coordination signal from the coordination unit and accomplish homologous task. Ultimately, the whole system could be controlled through the combined action of these sub-channels.

\section{MMCICS Design Inspired from NES}

\subsection{MMCICS Structure Design}

According to the bioinspired motion control approach, a novel multi-loop and multi-objective cooperative intelligent control system (MMCICS) is proposed to achieve intelligent coordination of position, velocity, and aceleration implemented by cooperation of several subchannels of plants, as shown in Figure 2. 


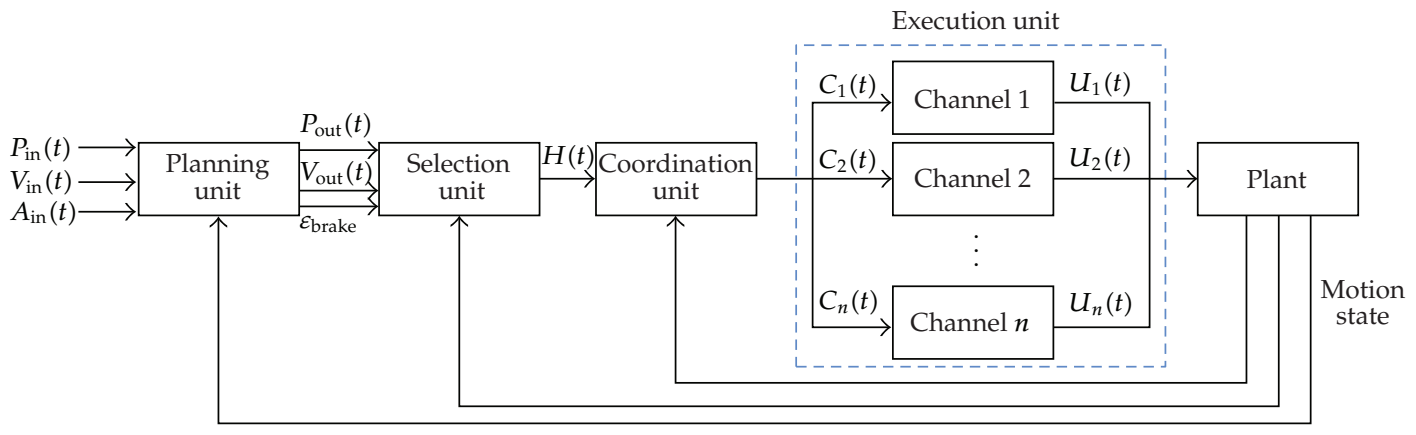

Figure 2: The structure of MMCICS.

\subsection{Units Design of MMCICS}

\subsubsection{Planning Unit}

The planning unit is primarily responsible for receiving and processing input signals of the position $P_{\text {in }}(t)$, the velocity $V_{\text {in }}(t)$, and the acceleration $A_{\text {in }}(t)$, and transmitting the desired position $P_{\text {out }}(t)$, the desired velocity $V_{\text {out }}(t)$, and the breaking factor $\varepsilon_{\text {brake }}$ signals to the selection unit. The planning algorithm includes the automatic braking process and the cooperative planning process.

(1) Automatic braking process. The position error is defined as

$$
e_{P 1}(t)=P_{\text {in }}(t)-P(t) .
$$

When it satisfies

$$
\left|e_{P 1}(t)\right| \leq \varepsilon_{\text {brake }}
$$

the input velocity signal is changed automatically to

$$
V_{\text {in }}(t)=0,
$$

where

$$
\varepsilon_{\text {brake }}=\left|\frac{V^{2}\left(t_{\text {brake }}\right)}{2 A_{\text {in }}\left(t_{\text {brake }}\right)}\right|
$$

is the braking factor, $t_{\text {brake }}$ is the initial time of the automatic braking process. The actual position signal $P(t)$ and the actual velocity signal $V(t)$ are obtained via ultralong feedback.

(2) Cooperative planning process. Since acceleration is hardly to be controlled directly, the $V_{\text {in }}(t)$ and the $A_{\text {in }}(t)$ are regulated by the cooperative planning process while the $P_{\text {in }}(t)$ is sent to the selection unit directly. Some typical planning methods have good 
results and have been used in practice for a long time. In order to test the control performance of the MMCICS more clearly, trapezoid curve method has been chosen in this paper. The algorithm can be described as

$$
\begin{gathered}
P_{\text {out }}(t)=P_{\text {in }}(t), \\
V_{\text {out }}(t)= \begin{cases}A_{\text {in }}(t) \cdot\left(t-t_{\text {up }}\right)+V\left(t_{\text {up }}\right), & t \in T_{\text {up }} \\
V_{\text {in }}(t), & t \in\left(T_{\text {up }} \cup T_{\text {down }}\right)^{c} \\
V\left(t_{\text {down }}\right)-A_{\text {in }}(t) \cdot\left(t-t_{\text {down }}\right), & t \in T_{\text {down }}\end{cases}
\end{gathered}
$$

where

$$
\begin{gathered}
T_{\text {up }}=\left\{t \mid A_{\text {in }}(t) \cdot\left(t-t_{\text {up }}\right)+V_{\text {out }}\left(t_{\text {up }}\right) \leq V_{\text {in }}\left(t_{\text {up }}\right)\right\}, \\
T_{\text {down }}=\left\{t \mid V_{\text {out }}\left(t_{\text {down }}\right)-A_{\text {in }}(t) \cdot\left(t-t_{\text {down }}\right) \geq V_{\text {in }}\left(t_{\text {down }}\right)\right\},
\end{gathered}
$$

where, $t_{\text {up }}$ and $t_{\text {down }}$ is the initial time when $V_{\text {in }}\left(t_{\text {up }}\right)>V_{\text {out }}\left(t_{\text {up }}\right)$ and $V_{\text {in }}\left(t_{\text {down }}\right)<$ $V_{\text {out }}\left(t_{\text {down }}\right)$, respectively.

\subsubsection{Selection Unit}

The selection unit is designed as a switcher for the real-time dominant control mode. This unit receives the actual position feedback signal via long-loop feedback mechanism while the dominant motion control signal is transmitted to the coordination unit. Velocity-velocity control mode is on when the actual position is far from desired position while velocity control signal is sent to keep smooth movement. Position-velocity control mode takes over when the actual position is close to the desired position while position control signal is send to achieve accurate position. This rule for automatic switching is described as follows $[6,20]$ :

$$
\text { strategy }= \begin{cases}\text { velocity-velocity, } & \mathbf{r}>\mathbf{r}_{\mathbf{c}} \\ \text { position-velocity, } & \mathbf{r} \leq \mathbf{r}_{\mathbf{c}}\end{cases}
$$

where $\mathbf{r}$ is the distance between actual position and desired position, and $\mathbf{r}_{\mathbf{c}}$ is a switcher distance which is decided by current state of plant and switching strategy. To guarantee smooth switch, a simple conversion factor $K_{c}$ is also designed in the selection unit. The control algorithm can be designed as follows:

$$
H(t)= \begin{cases}V_{\text {out }}(t), & \left|e_{P 2}(t)\right|>\varepsilon_{\text {brake }} \cdot \eta_{\text {switch }} \\ e_{P 2}(t) \cdot K_{c}, & \left|e_{P 2}(t)\right| \leq \varepsilon_{\text {brake }} \cdot \eta_{\text {switch }}\end{cases}
$$

where

$$
\begin{gathered}
e_{P 2}(t)=P_{\text {out }}(t)-P(t), \\
K_{c}=\frac{\left|V_{\text {out }}\left(t_{\text {switch }}\right)\right|}{\varepsilon_{\text {brake }} \cdot \eta_{\text {switch }}},
\end{gathered}
$$


where $H(t)$ is the output of the selection unit, $e_{P 2}(t)$ is the error signal between desired and actual position, $0 \%<\eta_{\text {switch }} \leq 100 \%$ is a switching coefficient which decides switching position, $K_{c}$ is the conversion factor, and $t_{\text {switch }}$ is the initial time of the switching process.

\subsubsection{Coordination Unit}

The coordination unit is a coordinator which sends cooperative control signals to each subchannel of the plant. Many methods and mathematic models are suitable for this unit, the velocity Jacobian matrix is chosen in this paper due to the velocity control is our foremost object. In this scenario, all the input signals and output signals are regarded as the velocity signals whether the velocity-velocity control mode or the position-velocity control mode is selected. That output signals can be calculated by

$$
\left[C_{1}(t), C_{2}(t), \ldots, C_{n}(t)\right]^{T}=J \cdot H(t)
$$

where $C_{i}(t)$ is the ouput signal of the coordination unit to channel $i,(i=1,2, \ldots, n)$ of the execution unit, $J$ is the velocity Jacobian matrix of the plant.

\subsubsection{Execution Unit}

The execution unit, which includes a number of sub-channels, is the core and key unit of the MMCICS. To keep sub-channels interact harmoniously and systematically, the same control method and control structure have been applied to each channel. As shown in Figure 3, each channel has its own independent control subsystem which includes a primary controller, a hormone regulation self-adaptive module (HRSM), and a controlled subpart of plant. There are two ultra-short loop feedbacks. One is that the actual velocity signal is fed back to the primary controller; the other is that the adjusted control parameters are fed back to the HRSM, which can improve the local and global control effectiveness.

Some advanced controllers widely used in industry can be applied as primary controller. The controller can obey PID control algorithm, fuzzy control algorithm [24, 25], H-infinity control algorithm [26, 27], and so forth. Due to their simpledescription, high-dependability, and satisfactory performances, in the MMCICS, the control law of primary controller obeys the conventional PID control algorithm

$$
O_{i}(t)=K p_{i}^{0} \cdot e_{i}(t)+K i_{i}^{0} \cdot \int e_{i}(t) d t+K d_{i}^{0} \cdot \frac{d e_{i}(t)}{d t}
$$

where

$$
e_{i}(t)=C_{i}(t)-v_{i}(t)
$$

is the error signal between the input signal $C_{i}(t)$ and the actual velocity $v_{i}(t)$ of the part $i$, $O_{i}(t)$ is the output of the primary controller, $K p_{i}^{0}, K i_{i}^{0}$, and $K d_{i}^{0}$ are the initial PID parameters.

The HRSM is designed to improve primary controller self-adaptive performance. The regulation algorithm of HRSM is inspired from hormone regulation mechanism which includes identification and regulation processes. 


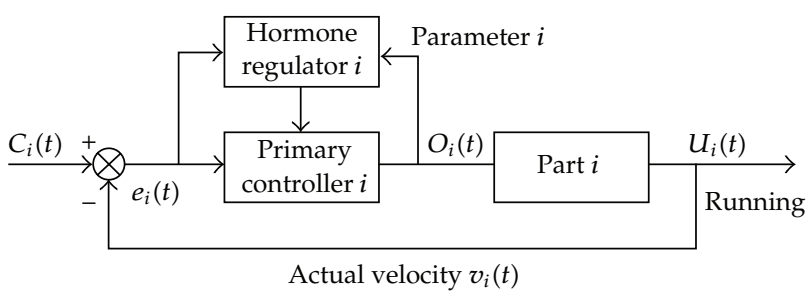

Figure 3: The structure of sub-channel.

(1) Identification. In NES, the gland can enhance identification and secretion precision within the working scope. However, when the stimulate signal beyond the control scope, hormone secretion rate is at its high limit. Similarly, the control error $e_{i}(t)$ in HRSM can be regarded as the stimulate signal, and its identification approach follows the principle of the hormone secretion. Therefore, the absolute value of control error $e_{i}(t)$ is calculated at first and then mapped to the correspond ding regulation scope. Hormone identification error $0 \leq E_{i}(t) \leq 1$ is defined as

$$
E_{i}(t)= \begin{cases}\frac{\left|e_{i}(t)\right|}{e_{i \max }-e_{i \min }}, & \left|e_{\mathrm{in}}(t)\right|<e_{i \max }-e_{i \min } \\ 1, & \left|e_{\mathrm{in}}(t)\right| \geq e_{i \max }-e_{i \min }\end{cases}
$$

where $e_{i \max }$ and $e_{i \min }$ are the high and low limited error of the optimal working scope, respectively.

(2) Regulation. The hormone secretion rate in NES is always nonnegative and monotone, and its secretion regulation mechanism usually follows the Hill functions, the growth curve, and so forth $[13,21]$. Based on the Sigmoid function, a hormone regulation factor is designed to regulate primary controller parameter as

$$
\alpha_{i}^{j}(t)=\frac{k_{i}^{j}}{1+\left(k_{i}^{j}-1\right) e^{-\beta_{i}^{j}\left(\left(E_{i}(t) / \eta_{i}^{j}\right)-1\right)}},
$$

where $j=p, i, d, 0 \%<\eta_{i}^{j} \leq 100 \%$ is the critical regulation coefficient, $k_{i}^{j} \geq 1$ is the high limited regulation coefficient, $0<\beta_{i}^{j} \leq 10$ is the sensitivity regulation coefficient. These three coefficients joint control the function curve's slope. Where $\eta_{i}^{j}$ decides the critical point between the up- and down-regulation, as

$$
\begin{array}{ll}
\alpha_{i}^{j}(t)<1, & E_{i}(t)<\eta_{i}^{j}, \\
\alpha_{i}^{j}(t)=1, & E_{i}(t)=\eta_{i}^{j}, \\
\alpha_{i}^{j}(t)>1, & E_{i}(t)>\eta_{i}^{j} .
\end{array}
$$


The $k_{i}^{j}$ decides the high limited value. Because if $\left(E_{i}(t) / \eta_{i}^{j}\right) \gg 1$, then $\mathrm{e}^{-\beta_{i}^{j}\left(\left(E_{i}(t) / \eta_{i}^{j}\right)-1\right)} \rightarrow 0$ that $\alpha_{i}^{j}(t) \rightarrow k_{i}^{j}$. Meanwhile, it also should be noted that if $k_{i}^{j}=1$, then $\alpha_{i}^{j}(t)=1$. The $\beta_{i}^{j}$ decides the response rate and has a major impact on the low limited value of $\alpha_{i}^{j}(t)$. When $\beta_{i}^{j}$ is bigger, the $\alpha_{i}^{j}(t)$ curve changes acutely and the low limit of $\alpha_{i}^{j}(t)$ is lower; in contrast, the gentle changes results to higher low limit.

Then primary controller parameter can be regulated by its control characteristic. In the PID control algorithm, when the control error is too big, the proportion gain $K p_{i}^{0}$ should decrease to weaken the control action, thus reduces the overshoot. In contrast, the proportion gain should increase to enhance control precision and eliminate control error quickly [20]. The correcting regulation of the integral coefficient $K i_{i}^{0}$ and the differential coefficient $K d_{i}^{0}$ are similar to that of the proportion gain. Therefore, the parameter regulation algorithm of the PID controller is

$$
\begin{aligned}
K p_{i}(t) & =K p_{i}^{0} / \alpha_{i}^{p}(t) K i_{i}(t) \\
& =K i_{i}^{0} \cdot \alpha_{i}^{i}(t) K d_{i}(t) \\
& =\frac{K d_{i}^{0}}{\alpha_{i}^{d}(t)} .
\end{aligned}
$$

where, when $\alpha_{i}^{p}(t)>1, K p_{i}^{0}$ will be reduced; when $\alpha_{i}^{p}(t)<1, K p_{i}^{0}$ will be increased; when $\alpha_{i}^{p}(t)=1, K p_{i}^{0}$ will not be changed. Meanwhile, $K i_{i}^{0}$ and $K d_{i}^{0}$ have similar regulation characteristics. The regulation principle of the HRSM satisfies the optimization task and then (3.11) will be changed to optimized control law

$$
O_{i}(t)=K p_{i}(t) \cdot e_{i}(t)+K i_{i}(t) \cdot \int e_{i}(t) d t+K d_{i}(t) \cdot \frac{d e_{i}(t)}{d t},
$$

where $K p_{i}(t), K i_{i}(t)$, and $K d_{i}(t)$ are optimized control parameters.

\subsection{Parameters Tuning of MMCICS}

(1) Tune the primary controller parameter. First, only take the primary controller into action, and then tune the initial control parameters $K p_{i}^{0}, K i_{i}^{0}$, and $K d_{i}^{0}$ approximately.

(2) Determine the high and low limited hormone identification error. According to the response characteristics of the experimental results in step (1), determine the high limited error $e_{i \max }$ and low limited error $e_{i \min }$ of the optimal working scope.

(3) Tune the regulation coefficients of the hormone regulator. Take the execution unit into action, according to the response characteristic and overshoot of the experimental results, tune the critical regulation coefficient $\eta_{i}^{j}$ to decide critical working point of the hormone regulator. And then when control error $e_{i}(t)$ is too big, tune the high limited regulation coefficient $k_{i}^{j}$ to ensure a stable and faster movement of the plant with little or without overshoot. In contrast, tune the sensitivity regulation coefficient $\beta_{i}^{j}$ to ensure accuracy and stability. 


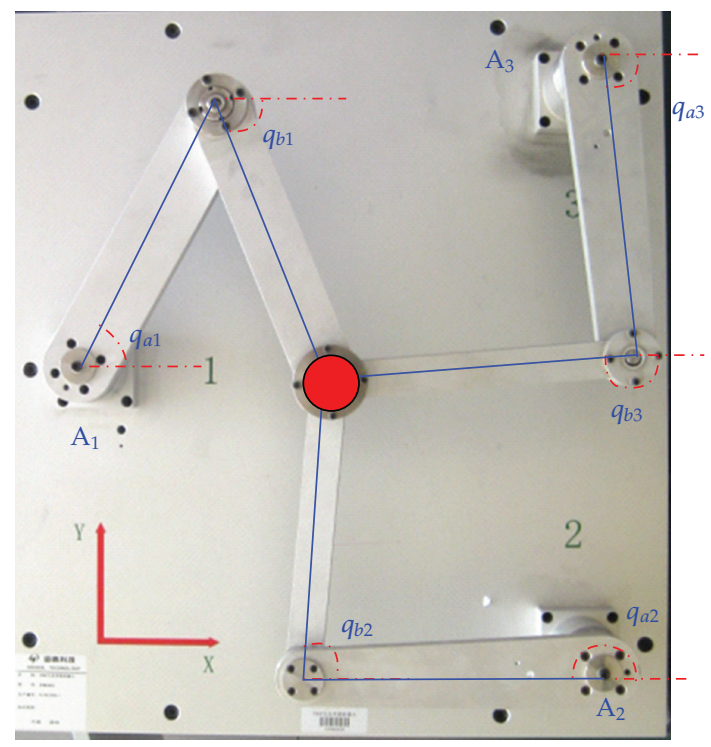

Figure 4: The 2-DOF redundant parallel manipulator.

(4) Determine the switching coefficient. Take the MMCICS into action and then determine the switching coefficient $\eta_{\text {switch }}$ to ensure the control strategy switching smoothly.

\section{Experimental Results and Analysis}

Some typical experimental results are provided in this section to explore two main experiments of proposed MMCICS. Firstly, the control results with and without HRSM are compared to find out whether HRSM yields better in subchannel experiment. Next more comprehensive experiments are performed to verify multiobject cooperative control performance of the MMCICS, and whether HRSM has better global control effect.

As shown in Figure 4, a 2-DOF redundant parallel manipulator (Googol Tech Ltd.'s GPM2002) $[28,29]$ is selected as the experiment platform due to its complex redundancy structure and multi-channel inputs. Three bases of the manipulator are equipped with three AC servo motors with harmonic gear drives. The coordinates of three bases are $A_{1}(0,250)$, $A_{2}(433,0)$, and $A_{3}(433,500)$, and all the links have the same length $l=244$. The unit of coordinates and length is millimeter. Active joint angles are $q_{a 1}, q_{a 2}$ and $q_{a 3}$, and passive joint angles are $q_{b 1}, q_{b 2}$ and $q_{b 3}$. Position signals of the motors are measured with the absolute optical electrical encoders, and input voltage signals are controlled by a motion control board. All algorithms are implemented with Matlab/Simulink environment on an industrial controlling computer with a $2.8 \mathrm{GHz}$ processor and $1024 \mathrm{MB}$ memory. The real-time implementation is executed with the Real Time Workshop (RTW) of Matlab, and sampling period is $5 \mathrm{~ms}$.

Firstly, to verify the effectiveness of the proposed HRSM in the execution unit, we only take active joint 1 (base $A_{1}$ ) without loads and links into action. The control performance of 
the conventional PID controller and the PID controller with HRSM (HRSM-PID) are compared under the six different velocities of the servo motor 1 , namely the motor of base $A_{1}$. To make the contrast effect more clearly, the conventional PID parameters are designed as the same as the initial PID parameters in HRSM-PID controller, as shown in Table 1.

Motor in sub-channel has different dynamic characteristics at different velocities but has similar results in the same parameter sets. Multiple experiments have the similar results, and a typical result is as shown in Figure 5(a), when motor is running at low velocities, the steady-state errors are obvious due to load influence. The HRSM-PID controller achieves better stabilities, higher accuracies, slightly faster dynamic responses, and lower or no overshoots, compared with the conventional PID controller. Figure 5(b) shows that when running at high velocities, the motor has better motion performance and spends more times to achieve higher velocity. HRSM-PID controller achieves significantly faster dynamic responses compared with the PID controller. Figure 5(c) shows a typical output control signal $O_{1}(t)$ when input velocity step is 5 . As the expected, when the error is too big, the HRSM decreases the output control signals to reduce the overshoot. In contrast, the output control signals are increased to enhance control precision and eliminate control error quickly. With such strong self- adaptability, the HRSM improves the dynamic performances. The detailed lower quartile, median, upper quartile, average, and variance of the 10 time's results are shown in Table 2. Where, $V_{d}$ is the desired velocity, $t_{s}$ is the settling time, $\sigma$ is the overshoot, and |ess| is the absolutely value of steady-state error. The sub-channel experimental results show that based on hormone regulation mechanism, the HRSM owns strong self-adaptability that improves the response, accuracy, and stability of the subchannel.

To verify the multiobject cooperative control performance of the MMCICS, the endeffector of the redundant parallel manipulator is viewed as a controlled plant, and three active joints are viewed as three subchannels. The velocity Jacobian matrix between the end-effector and three active joints is

$$
J=\frac{1}{l}\left(\begin{array}{cc}
\frac{\cos q_{b 1}}{\sin \left(q_{b 1}-q_{a 1}\right)} & \frac{\sin q_{b 1}}{\sin \left(q_{b 1}-q_{a 1}\right)} \\
\frac{\cos q_{b 2}}{\sin \left(q_{b 2}-q_{a 2}\right)} & \frac{\sin q_{b 2}}{\sin \left(q_{b 2}-q_{a 2}\right)} \\
\frac{\cos q_{b 3}}{\sin \left(q_{b 3}-q_{a 3}\right)} & \frac{\sin q_{b 3}}{\sin \left(q_{b 3}-q_{a 3}\right)}
\end{array}\right) .
$$

Due to the complex mechanism structure of the parallel manipulator with actuation redundancy, it is a typical nonlinear system and difficult to get the accurate dynamic and friction model $[28,29]$. Although the manipulator has different dynamic characteristics in different positions, velocities, and accelerations, the proposed MMCICS can overcome accurate mathematical model problem. To verify the control performance of the MMCICS more thoroughly and whether the HRSM also achieves better control effectiveness in the proposed MMCICS, many different experiments were tested and have similar results. A representative contrast experimental result is shown in Figure 6, where the MMCICS without HRSM is chosen as contrast control system (CCS). The experiments are implemented with the same input signals and control parameters. The starting position, input goal position, and input 


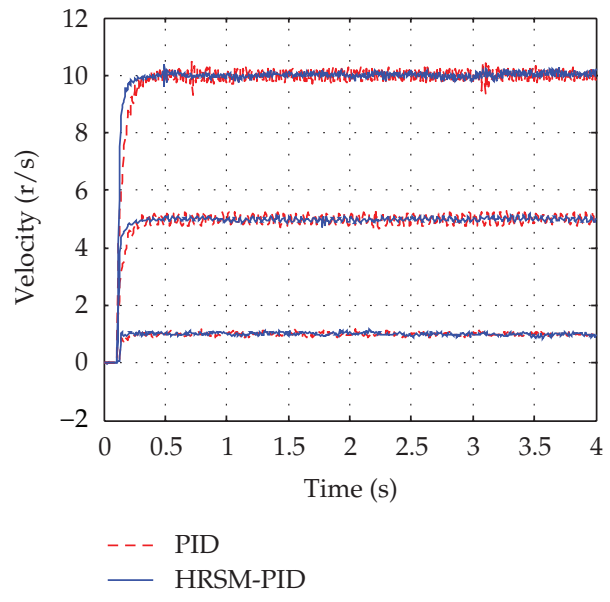

(a)

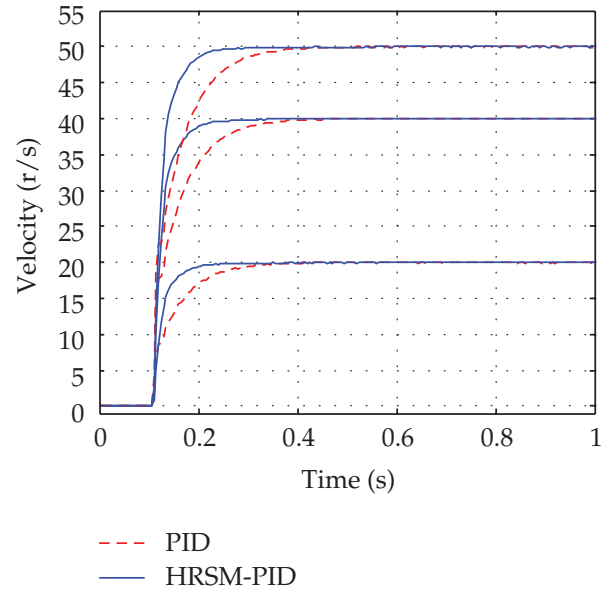

(b)



(c)

Figure 5: Contrast effect of the velocity control. (a) Low velocity control, (b) high velocity control. (c) Output control signal.

acceleration are $[216.5,250]^{T},[316.5,350]^{T}$, and $[1500,1500]^{T}$, respectively. The input velocity signal is

$$
\begin{aligned}
& V_{\text {in }}(t)=[0,0]^{T}, \quad t \geq 0 s \\
& V_{\text {in }}(t)=[100,100]^{T}, \quad t \geq 0.1 s \\
& V_{\text {in }}(t)=[300,300]^{T}, \quad t \geq 3.5 s .
\end{aligned}
$$

The switching coefficient is $\eta_{\text {switch }}=[20 \%, 20 \%]^{T}$ in selection unit, and control parameters in channel 1,2, and 3 are the same as in Table 1. Similarly, the parameters of CCS are the same as MMCICS.

As shown in Figures 6(a) and 6(b), MMCICS achieves a faster response, better stability, and higher accuracy of velocity control compared with CCS. Especially, when the manipulator is running at high velocities, it is hard to achieve object velocity using CCS, due to 


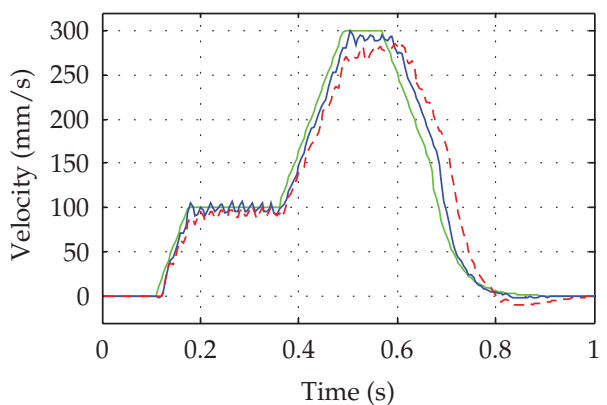

(a)

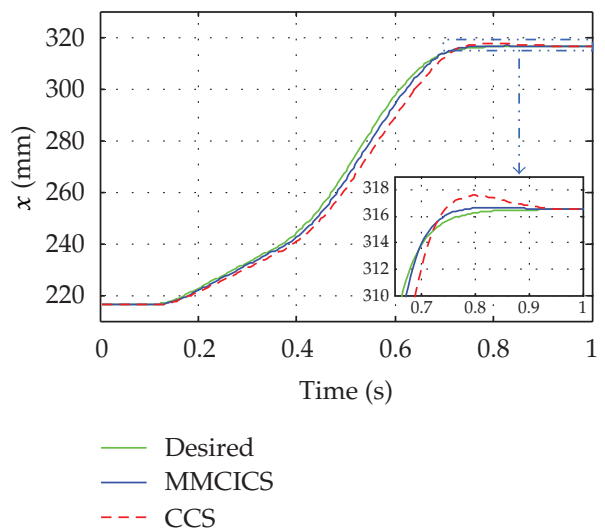

(c)

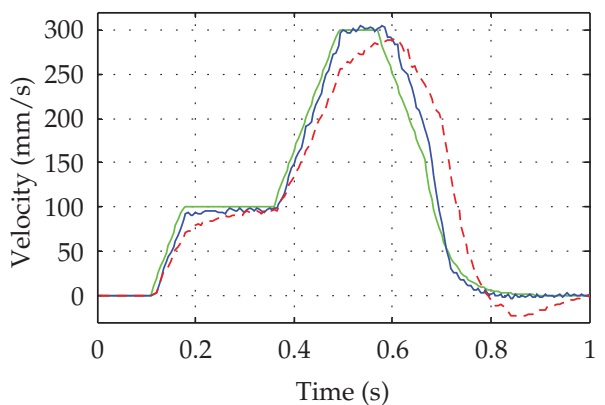

(b)

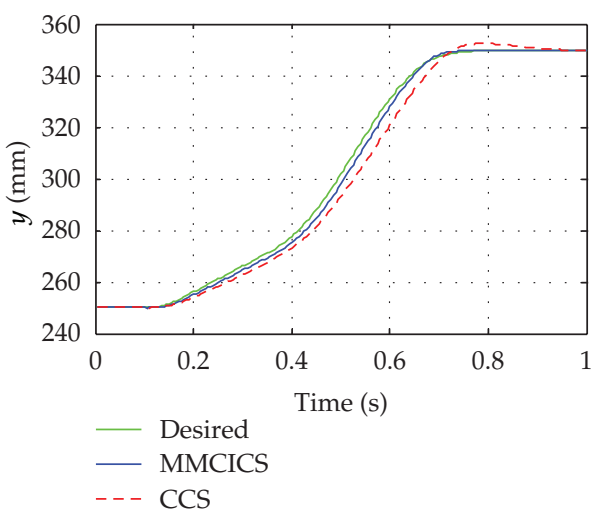

(d)

Figure 6: Multichannel control experimental results. (a) $X$-direction velocity. (b) $Y$-direction velocity. (c) $X$-direction position. (d) Y-direction position.

Table 1: Parameter set.

\begin{tabular}{lcc}
\hline Initial PID & Error factors & Hormone regulation factors \\
\hline$K p_{i}^{0}=0.03$ & $e_{i \max }=1 \mathrm{r} / \mathrm{s}$ & $k_{i}^{p}=5, \beta_{i}^{p}=1.2, \eta_{i}^{p}=20 \%$ \\
$K i_{i}^{0}=0.004$ & $e_{i \min }=-1 \mathrm{r} / \mathrm{s}$ & $k_{i}^{i}=5, \beta_{i}^{i}=1.2, \eta_{i}^{i}=15 \%$ \\
$K d_{i}^{0}=0.005$ & & $k_{i}^{d}=3, \beta_{i}^{d}=1, \eta_{i}^{d}=10 \%$ \\
\hline
\end{tabular}

complex plan structure and big load. However, MMCICS still maintains high performance as low velocity process. From the velocity response during the ascent, it's easy to find that the MMCICS has more stable acceleration response than CCS does. That means, based on the HRSM, the cooperative planning algorithm in the planning unit can be implemented easier for acceleration control. Moreover, during control strategy switching, CCS always has a significant negative overshoot of the velocity in braking process. In contrast, MMCICS can stop quickly with little or no negative overshoot due to its strong adaptability. Compared with Figures 6(a) and 6(b), we can find that, due to uneven distribution of loads, the CCS performance in $Y$-direction is worse than X-direction. However, MMCICS can overcome this problem, since its local self-adaptability improves the global self-adaptability.

Figures 6(c) and 6(d) shows that due to faster, more stable, and accurate velocity response, the MMCICS can achieve better position accuracy compared with the CCS. In the braking process, because of its better adaptability when control strategy is switched from 





Table 3: Performance evaluation for comprehensive experiment.

\begin{tabular}{lccccc}
\hline & \multicolumn{2}{c}{ Positon } & \multicolumn{2}{c}{ Veloctiy } & Error \\
& Final error & Settling time & \multicolumn{2}{c}{ Braking overshoot } \\
& & & $V_{\text {in }}(t)=[100,100]^{T}$ & $V_{\text {in }}(t)=[300,300]^{T}$ & \\
\hline MMCICS & {$[0.02,0.05]^{T}$} & $0.82 \mathrm{~s}$ & {$[6.62,7.50]^{T}$} & {$[7.22,5.64]^{T}$} & {$[2.41,3.13]^{T}$} \\
CCS & {$[0.05,0.22]^{T}$} & $1.07 \mathrm{~s}$ & {$[13.82,18.20]^{T}$} & {$[32.2,52.06]^{T}$} & {$[10.25,25.20]^{T}$} \\
\hline
\end{tabular}

the velocity-velocity control to the position-velocity control, the MMCICS has a faster position response, which makes position stable with lower overshoot or no overshoot.

Some compare results of the 10 time's average absolute values are shown in Table 3. The experimental results show that, with the planning algorithm in the planning unit, the soft switching algorithm in the selection unit, and the velocity cooperative control in the coordination unit, both MMCICS and CCS take advantages of position control and velocity control, and achieve cooperative control for position, velocity and acceleration. Particularly, with strong self-adaptability, faster response, and better stability of HRSM, control potentials of the MMCICS are exploited more thoroughly. The MMCICS achieves multi-objective cooperative intelligent control with higher performance even at high velocities and accelerations, for a nonlinear multi-input complex plant without accurate dynamics model.

\section{Conclusions}

This work presents a bioinspired cooperative intelligent control system for position, velocity, and acceleration multi-objective integrated control of a parallel plant. The similarity between the NES and motion control system revealed, and a bio-inspired motion control approach is proposed. Under the context of such approach, the MMCICS with system structure, algorithm, and steps in parameter tuning is proposed to achieve multiobjective control. The experiments are carried out with a 2-DOF redundant parallel manipulator where the feasibility of the new control system is demonstrated. The contrast effect shows that the stability, accuracy, adaptability, response rate of the proposed MMCICS is superior to those of the conventional controllers. According to our knowledge, this is the first time that NES-based MMCICS and HRSM are proposed and used for an actual parallel manipulator. The proposed MMCICS can be implemented easily and provides a new and efficient method for multiobjective integrated control of complex multichannel systems. In future works, force and torque control will be considered to establish a more complete multi-objective control system. More rigorous and advanced algorithm and proof are required instead of the PID controller. Besides, parameter optimization, dynamics, and stability analysis can be conducted on MMCICS.

\section{Acknowledgments}

This work was supported in part by the Key Project of the National Natural Science Foundation of China (No. 61134009), the National Natural Science Foundation of China (no. 60975059), Support Research Project of National ITER Program (no. 2010GB108004), Specialized Research Fund for the Doctoral Program of Higher Education from Ministry of Education of China (no. 20090075110002), Project of the Shanghai Committee of Science and Technology (Nos. 11XD1400100, 11JC1400200, 10JC1400200, and 10DZ0506500). 


\section{References}

[1] D. Zhang and Z. Gao, "Optimal kinematic calibration of parallel manipulators with pseudoerror theory and cooperative coevolutionary network," IEEE Transactions on Industrial Electronics, vol. 59, no. 8, pp. 3221-3231, 2012.

[2] H. Dong, Z. Wang, and H. Gao, "Distributed filtering for a class of time-varying systems over sensor networks with quantization errors and successive packet dropouts," IEEE Transactions on Signal Processing, vol. 60, no. 6, pp. 3164-3173, 2012.

[3] Z. Wang, H. Zeng, D. W. C. Ho, and H. Unbehauen, "Multiobjective control of a four-link flexible manipulator: a robust $\mathrm{H}_{\infty}$ approach," IEEE Transactions on Control Systems Technology, vol. 10, no. 6, pp. 866-875, 2002.

[4] B. Shen, Z. Wang, and X. Liu, "A stochastic sampled-data approach to distributed $\mathrm{H}_{\infty}$ filtering in sensor networks," IEEE Transactions on Circuits and Systems. I, vol. 58, no. 9, pp. 2237-2246, 2011.

[5] A. Khoukhi, "Data-driven multi-stage motion planning of parallel kinematic machines," IEEE Transactions on Control Systems Technology, vol. 18, no. 6, pp. 1381-1389, 2010.

[6] Z.Sun, R. Xing, C. Zhao, and W. Huang, "Fuzzy auto-tuning PID control of multiple joint robot driven by ultrasonic motors," Ultrasonics, vol. 46, no. 4, pp. 303-312, 2007.

[7] K. Pathak, J. Franch, and S. K. Agrawal, "Velocity and position control of a wheeled inverted pendulum by partial feedback linearization," IEEE Transactions on Robotics, vol. 21, no. 3, pp. 505-513, 2005.

[8] M. S. Tsai, H. W. Nien, and H. T. Yau, “Development of integrated acceleration/deceleration lookahead interpolation technique for multi-blocks NURBS curves," International Journal of Advanced Manufacturing Technology, vol. 56, no. 5-8, pp. 601-618, 2011.

[9] S. Mitra and Y. Hayashi, "Bioinformatics with soft computing," IEEE Transactions on Systems, Man, and Cybernetics Part A, vol. 36, no. 5, pp. 616-635, 2006.

[10] W. Savino and M. Dardenne, "Neuroendocrine control of thymus physiology," Endocrine Reviews, vol. 21, no. 4, pp. 412-443, 2000.

[11] E. B. Stear, "Application of control theory to endocrine regulation and control," Annals of Biomedical Engineering, vol. 3, no. 4, pp. 439-455, 1975.

[12] D. M. Keenan, J. Licinio, and J. D. Veldhuis, "A feedback-controlled ensemble model of the stressresponsive hypothalamo-pituitary-adrenal axis," Proceedings of the National Academy of Sciences of the United States of America, vol. 98, no. 7, pp. 4028-4033, 2001.

[13] L. S. Farhy, "Modeling of oscillations in endocrine networks with feedback," Methods in Enzymology, vol. 384, pp. 54-81, 2004.

[14] M. Neal and J. Timmis, "Timidity: a useful emotional mechanism for robot control?" Informatica, vol. 27, no. 2, pp. 197-204, 2003.

[15] P. Vargas, R. Moioli, L. N. d. Castro, J. Timmis, M. Neal, and F. J. V. Zuben, "Artificial homeostatic system: a novel approach," in Advances in Artificial Life, vol. 3630 of Lecture Notes in Computer Science, pp. 754-764, 2005.

[16] F. M. Córdova and L. R. Cañete, "The challenge of designing nervous and endocrine systems in robots," International Journal of Computers, Communications \& Control, vol. 1, no. 2, pp. 33-40, 2006.

[17] B. Liu, L. Ren, and Y. Ding, "A novel intelligent controller based on modulation of neuroendocrine system," in Proceedings of the 2nd International Symposium on Neural Networks: Advances in Neural Networks (ISNN'05), pp. 119-124, June 2005.

[18] Y. S. Ding and B. Liu, "An intelligent bi-cooperative decoupling control approach based on modulation mechanism of internal environment in body," IEEE Transactions on Control Systems Technology, vol. 19, no. 3, pp. 692-698, 2011.

[19] D. Tang, W. Gu, L. Wang, and K. Zheng, "A neuroendocrine-inspired approach for adaptive manufacturing system control," International Journal of Production Research, vol. 49, no. 5, pp. 1255-1268, 2011.

[20] C. Guo, K. Hao, Y. Ding, X. Liang, and Y. Dou, "A position-velocity cooperative intelligent controller based on the biological neuroendocrine system," in Advances in Neural Networks_ISNN 2011, vol. 6677 of Lecture Notes in Computer Science, pp. 112-121, 2011.

[21] C. E. Cortés, D. Sáez, F. Milla, A. Núñez, and M. Riquelme, "Hybrid predictive control for realtime optimization of public transport systems' operations based on evolutionary multi-objective optimization," Transportation Research Part C, vol. 18, no. 5, pp. 757-769, 2010.

[22] M. F. Prummel, L. J. S. Brokken, and W. M. Wiersinga, "Ultra short-loop feedback control of thyrotropin secretion," Thyroid, vol. 14, no. 10, pp. 825-829, 2004. 
[23] X. Liang, Y. S. Ding, L. H. Ren, K. R. Hao, H. P. Wang, and J. J. Chen, “A bioinspired multilayered intelligent cooperative controller for stretching process of fiber production," IEEE Transactions on Systems, Man and Cybernetics Part C, vol. 42, no. 3, pp. 367-377, 2012.

[24] R. E. Precupa and H. Hellendoornb, "A survey on industrial applications of fuzzy control," Computers in Industry, vol. 62, no. 3, pp. 213-226, 2011.

[25] H. Dong, Z. Wang, D. W. C. Ho, and H. Gao, "Robust $\mathrm{H}_{\infty}$ fuzzy output-feedback control with multiple probabilistic delays and multiple missing measurements," IEEE Transactions on Fuzzy Systems, vol. 18, no. 4, pp. 712-725, 2010.

[26] Z. Wang, B. Shen, H. Shu, and G. Wei, “Quantized $\mathrm{H}_{\infty}$ control for nonlinear stochastic time-delay systems with missing measurements," IEEE Transactions on Automatic Control, vol. 7, no. 6, pp. 1431-1444, 2012.

[27] Z. Wang, B. Shen, and X. Liu, " $\mathrm{H}_{\infty}$ filtering with randomly occurring sensor saturations and missing measurements," Automatica, vol. 48, no. 3, pp. 556-562, 2012.

[28] W. Shang and S. Cong, "Nonlinear computed torque control for a high-speed planar parallel manipulator," Mechatronics, vol. 19, no. 6, pp. 987-992, 2009.

[29] W. Shang, S. Cong, and F. Kong, "Identification of dynamic and friction parameters of a parallel manipulator with actuation redundancy," Mechatronics, vol. 20, no. 2, pp. 192-200, 2010. 


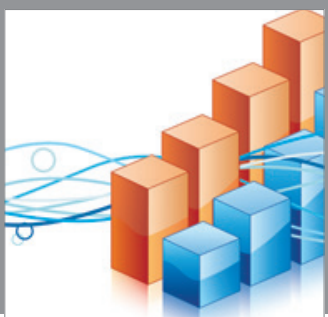

Advances in

Operations Research

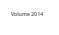

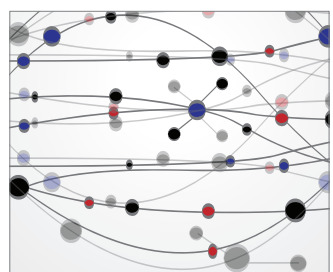

\section{The Scientific} World Journal
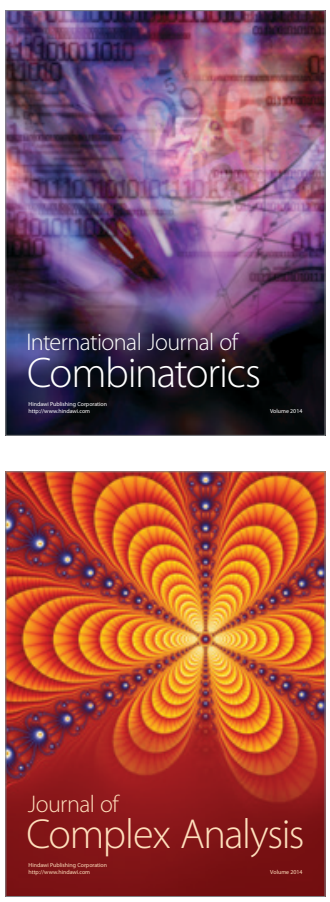

International Journal of

Mathematics and

Mathematical

Sciences
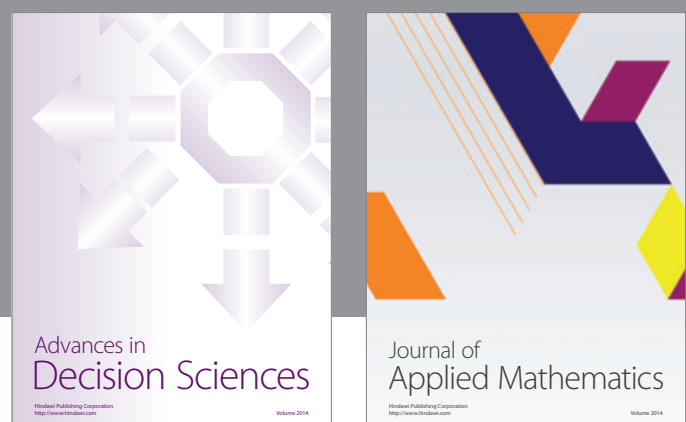

Journal of

Applied Mathematics
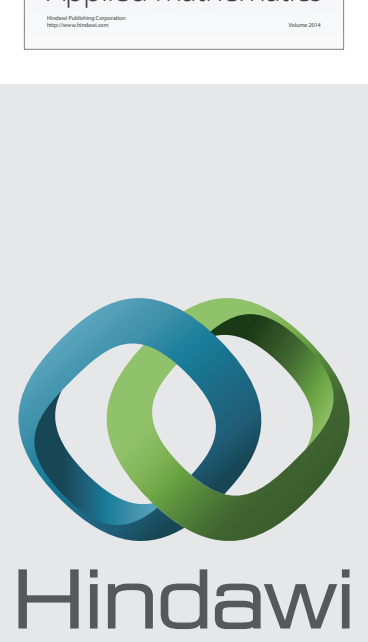

Submit your manuscripts at http://www.hindawi.com
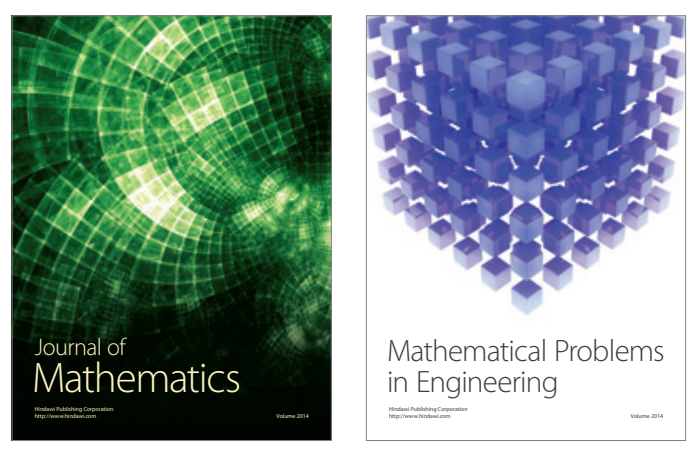

Mathematical Problems in Engineering
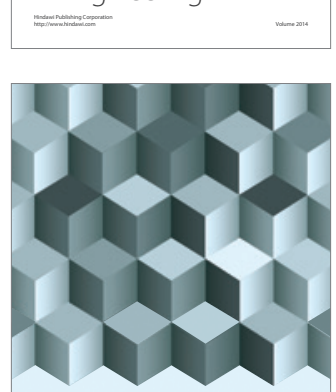

Journal of

Function Spaces
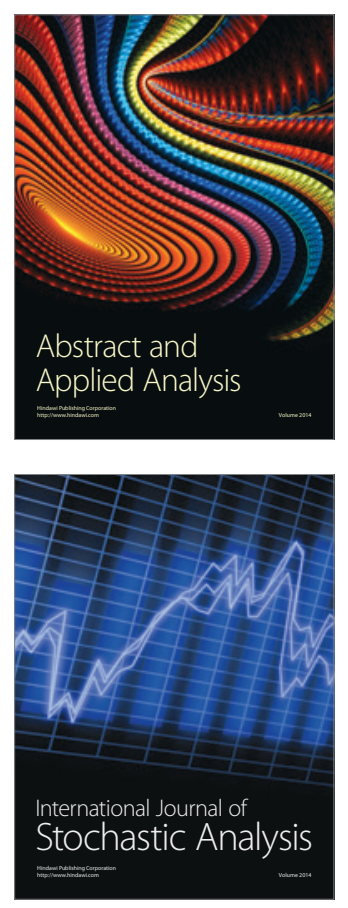



ournal of

Probability and Statistics

Promensencen
